\title{
Cardiac MRI: T2-Mapping Versus T2-Weighted Dark-Blood TSE Imaging for Myocardial Edema Visualization in Acute Myocardial Infarction
}

\section{Kardiales MRT: T2-Mapping versus T2-gewichtete TSE-Bildgebung für die Visualisierung eines Myokardödems beim akuten Myokardinfarkt}

Authors

Affiliations
K. Naßenstein ${ }^{1}$, F. Nensa' ${ }^{1}$, T. Schlosser ${ }^{1}$, O. Bruder ${ }^{2}$, L. Umutlu' ${ }^{1}$, T. Lauenstein ${ }^{1}$, S. Maderwald ${ }^{3}$, M. E. Ladd ${ }^{3}$

Dep. of Diagnostic and Interventional Radiology and Neuroradiology, University Hospital Essen

Department of Cardiology and Angiology, Contilia Heart and Vascular Center, Elisabeth Hospital Essen

Erwin L. Hahn Institute for Magnetic Resonance Imaging, University Duisburg-Essen

\author{
Key words \\ - heart \\ - MR imaging \\ - T2 mapping \\ - myocardial infarction
}

received $\quad 14.3 .2013$

accepted 19.7.2013

\section{Bibliography}

DOI http://dx.doi.org/

10.1055/s-0033-1350516

Published online: 30.9.2013

Fortschr Röntgenstr 2014; 186 :

166-172 @ Georg Thieme

Verlag KG Stuttgart · New York . ISSN 1438-9029

\section{Correspondence \\ Dr. Kai Naßenstein}

Institut für Diagnostische und Interventionelle Radiologie und Neuroradiologie, Universitätsklinkum Essen Hufelandstraße 55

45122 Essen

Germany

Tel.: ++49/0201/7232311

Fax: ++49/02 01/7231548

Kai.Nassenstein@uni-due.de

\section{Zusammenfassung}

$\nabla$

Ziel: Ziel der vorliegenden Studie war es, zu untersuchen mit welcher diagnostischen Genauigkeit das T2-Mapping die Visualisierung eines Myokardödems beim akuten Myokardinfarkt (AMI) erlaubt und diese diagnostische Genauigkeit mit der des gegenwärtigen Standards für die myokardiale Ödembildgebung (T2-gewichtete, dark-blood präparierte TSE-Sequenz) zu vergleichen.

Material und Methoden: 29 Patienten mit AMI wurde bei 1,5 T untersucht. Zur Ödemdarstellung wurden T2-Maps, welche aus jeweils 3 unterschiedlich T2-gewichteten SSFP-Bildern berechnet wurden, sowie T2-gewichtete dark-blood TSE Bilder in Standardkurz- und Langachsenschichten akquiriert. Zur Analyse der regionalen linksventrikulären (LV) Funktion wurden cine SSFP, zur Darstellung der Myokardnekrosen Late Gadolinium Enhancement (LGE) Bilder akquiriert. Die T2-Maps sowie die T2w-dark-blood-TSE-Datensätze wurden zweimal unabhängig von den übrigen Sequenzen visuell ausgewertet, wobei pro LVSegment analysiert wurde ob ein Ödem sichtbar ist oder nicht. Basierend auf den cine SSFP und LGE-Bildern wurde die Infarktzone als Referenzstandard festgelegt.

Ergebnisse: Das T2-Mapping zeigte in dieser segmentbasierten Analyse eine Sensitivität von $82 \%$ und eine Spezifität von $94 \%$ für die Detektion eines Ödems in der Infarktzone; die T2w-dark-bloodTSE-Sequenz eine Sensitivität von $50 \%$ und einer Spezifität von $98 \%$. Das T2-Mapping zeigte im Vergleich zur T2w-dark-blood-TSE-Sequenz eine höhere Interrater-Reliabilität ( $\mathrm{k:} 0,87$ vs. 0,76).

Schlussfolgerung: Das T2-Mapping erlaubt die Visualisierung des Myokardödems beim AMI mit hoher Sensitivität und Spezifität und weist aufgrund einer höheren Sensitivität eine bessere diagnostische Genauigkeit als die T2w-dark-bloodTSE-Sequenz auf.

\section{Abstract \\ V}

Purpose: To assess the diagnostic accuracy of T2 mapping for the detection of myocardial edema in acute myocardial infarction (AMI), and to compare this diagnostic accuracy with that of the current standard for myocardial edema imaging, which is T2w dark-blood TSE imaging.

Materials and Methods: 29 patients with AMI were examined at $1.5 \mathrm{~T}$. For the visualization of myocardial edema, T2 maps, calculated from three T2w SSFP images, and T2w dark-blood TSE images were acquired in standard short- and long-axis views. Cine SSFP images were acquired for the analysis of left ventricular (LV) function and late gadolinium enhancement images (LGE) for the visualization of myocardial necrosis. The T2 maps as well as the T2w dark-blood TSE images were evaluated twice independently from the cine SSFP and LGE images. The presence or absence of myocardial edema was rated visually for each LV segment. As the standard of reference, the infarct zone was defined based on the cine SSFP and the LGE images. Results: In this segment-based analysis, T2 mapping showed a sensitivity of $82 \%$ and a specificity of $94 \%$ for the detection of edema in the infarct zone. T2w dark-blood TSE imaging revealed a sensitivity of $50 \%$ and a specificity of $98 \%$. T2 mapping showed a higher intra-rater agreement compared to T2 $\mathrm{w}$ dark-blood TSE imaging ( $\mathrm{k}$ : 0.87 vs. 0.76 ).

Conclusions: T2 mapping allows for the visualization of myocardial edema in AMI with a high sensitivity and specificity, and features better diagnostic accuracy in terms of a higher sensitivity compared to T2w dark-blood TSE imaging.

Citation Format:

- Naßenstein K, Nensa F, Schlosser T et al. Cardiac MRI: T2-Mapping Versus T2-Weighted DarkBlood TSE Imaging for Myocardial Edema Visualization in Acute Myocardial Infarction. Fortschr Röntgenstr 2014; 186: 166-172 


\section{Introduction}

Within the last decade, cardiac magnetic resonance imaging (CMR) has become an important diagnostic tool for the assessment of acute myocardial infarction (AMI) because it allows the visualization and quantification of myocardial necrosis and microvascular obstruction as well as the analysis and quantification of global and regional left ventricular (LV) function within a single examination [1-4]. Beyond the assessment of these important prognostic parameters, CMR allows the visualization of myocardial edema by the acquisition of T2-weighted images [5]. The detection of myocardial edema in the setting of myocardial ischemia is important since it permits the differentiation between areas of acute and chronic myocardial infarction [6], and, moreover, the determination of the area at risk of infarction [7].

Currently, T2-weighted turbo-spin-echo (TSE) sequences with dark-blood preparation are the standard for myocardial edema imaging in the clinical routine [8, 9]. Unfortunately, T2-weighted dark-blood TSE sequences are prone to image artifacts such as signal intensity variations caused by the use of phased-array coils, bright rim artifacts next to the endocardium caused by insufficient suppression of the signal from slow flowing blood, or posterior wall signal loss caused by through-plane motion of the dark-blood prepared slice out of the imaging slice in the case of improper timing [10-12]. Consequently, Kellman et al. showed that T2-weighted dark-blood TSE imaging was either non-diagnostic or incorrect in $29 \%$ of patients with AMI [13].

Recently, a quantitative T2 mapping technique that overcomes these limitations has been introduced for the evaluation of myocardial edema [14]. In short, this technique is based on the acquisition of three T2-prepared steady state free precession (SSFP) images, each acquired with a different $\mathrm{T} 2$ preparation time. Since image acquisition is done in the transient mode of single-shot SSFP immediately after T2 preparation, the signal in each image is dominated by the T2 preparation and, therefore, represents a different echo time along the T2 decay curve, thus enabling the calculation of the T2 relaxation times of each image pixel [14]. In a first clinical study, Verhaert et al. recorded the myocardial T2 values prospectively in 16 myocardial segments in 27 patients admitted with AMI and demonstrated that the average T2 values are higher in the infarct zone compared to remote myocardium [15]. Since in this study the infarct zone had been defined independently from the $\mathrm{T} 2$ maps based on the analysis of cine images and late gadolinium enhancement (LGE) images, and the T2 values of the infarct zone and remote myocardium had been calculated, thereafter, based on these predefined areas, the study of Verhaert et al. does not allow a statement concerning the diagnostic accuracy of T2 mapping independent of other sequences (e.g. cine images, LGE) [15]. However, beyond the quantification of T2 values in predefined areas, an essential requirement for edema imaging is that it enables reliable determination between edematous and non-edematous myocardium independent of other sequences (e.g. for diagnosis of acute myocarditis [9]). Therefore, our study aimed to analyze the accuracy of T2 mapping for visual myocardial edema detection on a segmental basis in patients with AMI in comparison to the current standard for myocardial edema imaging, which is T2-weighted TSE imaging with dark blood preparation $[8,9]$.

\section{Subjects and Methods \\ $\nabla$}

The study was approved by the local ethics committee, and written informed consent was obtained from all patients prior to CMR. Patients with a first AMI as defined by established diagnostic criteria [16] were consecutively enrolled. Both ST-segment elevation myocardial infarction (STEMI) and non-ST-segment elevation myocardial infarction (NSTEMI) were considered eligible. Patients with impaired renal function defined as a glomerular filtration rate (GFR) $<60 \mathrm{ml} / \mathrm{min} / 1.73 \mathrm{~m}^{2}$ were excluded from the study as well as patients with a known history of chronic myocardial infarction or general contraindications for magnetic resonance imaging (MRI).

During a period of 3 months, 29 consecutive patients (22 men, 7 women, mean age $59 \pm 11$ years) with a first AMI, who were willing to participate in the present study, were enrolled. 7 further patients ( 5 men, 2 women, mean age $63 \pm 9$ years), who were considered to be eligible for the present study, refused study participation. The baseline characteristics of the enrolled study population are given in $\bullet$ Table 1 in detail.

\section{Image acquisition}

MR examinations were performed on a 1.5 Tesla MR scanner (Magnetom Avanto, Siemens Healthcare, Erlangen, Germany) equipped with high-performance gradients (SQ-Engine: maximum amplitude $45 \mathrm{mT} / \mathrm{m}$, slew rate $200 \mathrm{mT} / \mathrm{m} / \mathrm{ms}$ ). For image

Table 1 Baseline characteristics of the study population $(n=29)$.

Tab. 1 Basischarakteristika der Studienpopulation $(n=29)$.

\begin{tabular}{|c|c|}
\hline male/female & $(76 \%) / 7(24 \%)$ \\
\hline age & $\begin{array}{l}59 \pm 11 \text { years; range } 40-77 \\
\text { years }\end{array}$ \\
\hline \multicolumn{2}{|l|}{ cardiovascular risk factors } \\
\hline hypertension & $23(79 \%)$ \\
\hline hyperlipidemia & $13(45 \%)$ \\
\hline hypercholesterolemia & $6(21 \%)$ \\
\hline smoking history: current/former/never & $12(41 \%) / 5(17 \%) / 12(41 \%)$ \\
\hline diabetes & $4(14 \%)$ \\
\hline obesity & $7(24 \%)$ \\
\hline previous myocardial infarction & $0(0 \%)$ \\
\hline previous CABG & $0(0 \%)$ \\
\hline previous $\mathrm{PCl}$ & $0(0 \%)$ \\
\hline STEMI/NSTEMI & $26(90 \%) / 3(10 \%)$ \\
\hline \multicolumn{2}{|l|}{ laboratory diagnostics } \\
\hline peak troponin $\mathrm{T}$ & median: 2.0; IQR: 2.89 \\
\hline creatine kinase (CK) & median: 971.0; IQR: 1650.75 \\
\hline creatine kinase MB (CK-MB) & median: 116.0; IQR: 174.25 \\
\hline \multicolumn{2}{|l|}{ culprit coronary artery } \\
\hline left anterior descending & $15(52 \%)$ \\
\hline circumflex artery & $5(17 \%)$ \\
\hline right coronary artery & $9(31 \%)$ \\
\hline \multicolumn{2}{|l|}{ revascularization } \\
\hline $\mathrm{PCl}$ & $28(97 \%)$ \\
\hline CABG & $0(0 \%)$ \\
\hline none & $1(3 \%)$ \\
\hline $\begin{array}{l}\text { time between onset of symptoms and re- } \\
\text { vascularization }\end{array}$ & $\begin{array}{l}\text { median: } 5.0 \text { hours ; IQR: } 5.9 \\
\text { hours; range } 1.0 \text { - } 149 \text { hours }\end{array}$ \\
\hline time between onset of symptoms and CMR & $\begin{array}{l}4.1 \pm 2.8 \text { days; range } \\
1-10 \text { days }\end{array}$ \\
\hline
\end{tabular}

Abbreviations: STEMI = ST-segment elevation myocardial infarction, NSTEMI = non-STsegment elevation myocardial infarction, $\mathrm{CABG}$ = coronary artery bypass grafting, $\mathrm{PCl}=$ percutaneous coronary intervention, $\mathrm{CMR}=$ cardiac magnetic resonance imaging, IQR interquartile range. 
acquisition, 6 elements of the spine matrix coil and all 6 elements of a phased-array torso coil (Tim Body Matrix Coil, Siemens Healthcare, Erlangen, Germany) were used.

A T2-weighted double inversion recovery (DIR) dark-blood prepared ECG-triggered, GRAPPA (GeneRalized Autocalibrating Partially Parallel Acquisition) accelerated, turbo-spin-echo (T2w DIR-TSE) sequence (echo time (TE) $74 \mathrm{~ms}$, repetition time (TR) $2 \times R R$, angle of excitation (EA) $180^{\circ}$, echo train length 17 , bandwidth $235 \mathrm{~Hz} /$ pixel, field of view (FOV) $244 \times 300 \mathrm{~mm}^{2}$ to $325 \times 400 \mathrm{~mm}^{2}$ depending on the anatomy of the individual patient, matrix $156 \times 256$, slice thickness $6 \mathrm{~mm}$, acceleration factor 2 ) was acquired in standard short and long-axis orientation to visualize myocardial edema. To compensate coil sensitivity variations, the standard manufacturer's coil sensitivity normalization (prescan normalization filter) was used.

For T2 mapping, three differently T2-prepared, GRAPPA accelerated, steady state free precession (SSFP) images (TR $3 \times$ RR, FA 70 ${ }^{\circ}$, echo spacing $2.5 \mathrm{~ms}$, T2-preparation times (T2 p) $0 \mathrm{~ms} / 24 \mathrm{~ms} /$ $55 \mathrm{~ms}$, FOV $244 \times 300 \mathrm{~mm}^{2}$ to $325 \times 400 \mathrm{~mm}^{2}$ depending on the anatomy of the individual patient, matrix $104 \times 160$, bandwidth $947 \mathrm{~Hz} /$ pixel, slice thickness $6 \mathrm{~mm}$, acceleration factor 2) were acquired in single-shot mode within one breath-hold per slice position. Although the sequence used ensures that all three images are acquired at the same point in the cardiac cycle, a non-rigid motion correction algorithm was used to adjust for moderate inplane misregistration between the three images that may be caused by differences in cardiac cycle length or by a slight diaphragm position drift. From the three motion-corrected images, the T2 maps were automatically generated based on a linear 2-parameter model after logarithmic transformation, as previously described [14]. T2 maps were acquired in standard short and long-axis orientation similar to the T2w DIR-TSE images.

A retrospectively ECG-gated, GRAPPA accelerated, SSFP sequence (TR $2.6 \mathrm{~ms}$, TE $1.1 \mathrm{~ms}$, EA $60^{\circ}$, FOV $244 \times 300 \mathrm{~mm}^{2}$ to $325 \times 400 \mathrm{~mm}^{2}$ depending on the anatomy of the individual patient, matrix $156 \times 192$, acquisition of $15 \mathrm{k}$-space lines per segment resulting in a temporal resolution of $39.5 \mathrm{~ms}, 25$ calculated phases, bandwidth $930 \mathrm{~Hz} /$ pixel, slice thickness $6 \mathrm{~mm}$, acceleration factor 2) was acquired in standard short and long-axis orientation for global and regional LV function analysis.

Inversion recovery (IR) spoiled-gradient-echo (GE) images (TR $8 \mathrm{~ms}$, TE $4 \mathrm{~ms}$, FA $30^{\circ}$, FOV $244 \times 300 \mathrm{~mm}^{2}$ to $325 \times 400 \mathrm{~mm}^{2}$ depending on the anatomy of the individual patient, matrix $172 \times 256$, slice thickness $6 \mathrm{~mm}$, bandwidth $140 \mathrm{~Hz} /$ pixel) were acquired $10-15$ minutes after intravenous injection of $0.2 \mathrm{mmol}$ per kg body weight Gd-DTPA (Magnevist, Bayer Schering Pharma AG, Berlin, Germany) in standard short and long-axis views to visualize myocardial necrosis [17]. For each scan the optimal inversion time (TI) to null the signal of normal myocardium was determined by using an SSFP sequence with incrementally increasing inversion times (TE $1.2 \mathrm{~ms}$, TR $2.4 \mathrm{~ms}$, EA 50 ${ }^{\circ}$, FOV $244 \times 300 \mathrm{~mm}^{2}$ to $325 \times 400 \mathrm{~mm}^{2}$ depending on the anatomy of the individual patient, matrix $78 \times 192$, slice thickness $8 \mathrm{~mm}$, bandwidth $965 \mathrm{~Hz} /$ pixel, increasing inversion times (TI) in steps of $27.5 \mathrm{~ms}$ ).

\section{Image analysis}

The T2w DIR-TSE datasets as well as the T2 maps were evaluated twice by one experienced observer (who had previously evaluated $>5000 \mathrm{CMR}$ ) in randomized order without access to the other acquired sequences. A minimum time interval of 14 days was maintained between the two evaluations to avoid a systema- tic bias. For semi-quantitative assessment of the extent of myocardial edema, an approach was used that had been described previously [18]. Based on the American Heart Association (AHA) segmental model of the left ventricle, the extent of edema was visually assessed for each LV segment, with the exception of segment 17 (apex), based on a 5-point scale (0: no edema; 1: 1 - 25\% edematous myocardium; $2: 26 \%$ to $50 \%$ edematous myocardium; 3: 51 - $75 \%$ edematous myocardium; $4: 76 \%$ to $100 \%$ edematous myocardium) for the T2 maps and T2w DIR-TSE datasets, and the segmental edema scores were summed to yield aggregate edema scores for each patient (possible range of the aggregate score: 0 (no edema visible in the entire LV myocardium) to 64 (edema of the entire LV myocardium). The image quality of T2w DIR-TSE images as well as of the T2 maps was visually analyzed based on a binary assessment (presence vs. absence of image artifacts that hamper the diagnostic value), and the number of segments with non-diagnostic image quality was assessed for both edema imaging approaches.

A second experienced observer (who had previously evaluated $>4000$ CMR), blinded to the edema imaging results, reviewed the cine and late gadolinium enhancement (LGE) images. Regional wall motion was visually evaluated and characterized as normal, hypokinetic, akinetic or dyskinetic for all cardiac segments. The extent of LGE within each myocardial segment was rated analog to the segmental assessment of myocardial edema, and similarly the segmental LGE scores were summed to yield an aggregate LGE score for each patient. As the standard of reference, the infarct zone was defined based on the cine and LGE images as well as on pertinent clinical information including coronary angiograms using the AHA 17-segment model of the left ventricle, whereby segment 17 (LV apex) was excluded from analysis. A segment of the LV was considered to be affected by acute ischemia if it belonged to the perfusion territory of the culprit coronary artery and showed an LGE in an ischemic pattern and/or a wall motion abnormality. Calculations of LV volumes and mass were performed based on the short-axis scans using ARGUS ${ }^{\mathrm{TM}}$ software (Siemens Healthcare, Erlangen, Germany) with semiautomatic contour detection. Manual correction of the automatically rendered contours was performed in all cases. Papillary muscles and myocardial trabeculations were included into the ventricular cavity. End-diastole was defined visually as the phase of the cardiac cycle with the largest LV cavity at mid-ventricle, and end-systole as the phase with the smallest LV cavity. At the base of the heart, slices were considered to be in the left ventricle if at least $270^{\circ}$ of the blood was surrounded by myocardium. The apical slice was defined as the last slice showing intra-cavity blood pool.

\section{Statistical analysis}

Statistical analysis was performed using MedCalc Version 11.4.2.0 (MedCalc Software, Mariakerke, Belgium). Continuous data with normal distribution are given as mean \pm standard deviation, nonnormally distributed data as median and inter-quartile range. Categorical data are expressed as frequency or percentage. The sensitivity, specificity, positive predictive values as well as negative predictive values were calculated for T2 mapping as well as for T2w DIR-TSE imaging. For statistical analysis, a two-tailed t-test for paired samples was used, or a Wilcoxon test in the case of non-normally distributed data. Cohen's kappa coefficients were calculated for statistical analysis of the intra-rater reliability. 


\section{Results}

\section{Standard of reference: Definition of the infarct zone}

Wall motion analysis revealed hypokinesia in 92 , akinesia in 28 , and dyskinesia in $3 \mathrm{LV}$ segments resulting in a total of $123 \mathrm{LV}$ segments with regional wall motion abnormalities. Assessment of LV volumes and global LV function revealed an end-diastolic volume of $133.6 \pm 47.1 \mathrm{ml}$, an end-systolic volume of $64.6 \pm 31.7 \mathrm{ml}$, a stroke volume of $69.0 \pm 22.4 \mathrm{ml}$, and an ejection fraction of 53.0 $\pm 10.5 \%$. In 132 LV segments, myocardial necrosis was observed by LGE imaging, and a mean LGE score of $15.3 \pm 7.0$ (range 2 to 32 ) was observed. From the analysis of the cine and LGE images and the coronary angiograms, 139 LV segments were rated as affected and 325 as non-affected by AMI.

\section{T2 mapping}

T2 mapping revealed myocardial edema within the infarct zone in all patients $(100 \%)$ during the first blinded evaluation of the T2 maps and in 28 patients (96.6\%) during the second image analysis. Combining both blinded evaluations resulted in a sensitivity of $82.0 \%$, a specificity of $94.0 \%$, a positive predictive value of $85.4 \%$, and a negative predictive value of $92.4 \%$ for the segment-based detection of myocardial edema within the infarct zone. Detailed results of the blinded reading are given in - Table 2. Statistical analyses showed a very good intra-rater agreement concerning the segmental rating of myocardial segments as edematous or non-edematous ( $k=0.865$; $95 \%$ confidence interval 0.815 to 0.915 ). Combining both blinded evaluations resulted in a mean edema score of $17.8 \pm 8.5$. Statistical analysis showed no significant differences between the T2 map edema scores and the observed LGE score ( $17.7 \pm 8.0$ vs. $15.3 \pm 7.0$, $p=0.091 ; 17.8 \pm 9.1$ vs. $15.3 \pm 7.0, p=0.134$ for the first and second evaluations, respectively). Image artifacts were observed in 3 of 369 (0.8\%) acquired T2 maps. However, all LV segments were rated as evaluable.

Table 2 Results of the blinded reading of the T2 mapping and T2w DIR-TSE datasets.

Tab. 2 Ergebnisse der verblindeten Auswertung der T2-Mapping sowie T2-gewichteten DIR-TSE-Datensätze.

\begin{tabular}{|lllll}
\hline & T2 mapping & T2w DIR-TSE \\
\hline & $\begin{array}{l}\mathbf{1}^{\text {st }} \text { blinded } \\
\text { reading }\end{array}$ & $\begin{array}{l}\mathbf{2}^{\text {nd }} \text { blinded } \\
\text { reading }\end{array}$ & $\begin{array}{l}\mathbf{1}^{\text {st }} \text { blinded } \\
\text { reading }\end{array}$ & $\begin{array}{l}\mathbf{2}^{\text {nd }} \text { blinded } \\
\text { reading }\end{array}$ \\
\hline LV segments rated as & & & & \\
\hline edematous & 131 & 134 & 78 & 76 \\
\hline non-edematous & 333 & 330 & 386 & 388 \\
\hline edema score & $17.7 \pm 8.0$ & $17.8 \pm 9.1$ & $9.6 \pm 7.6$ & $9.7 \pm 7.8$ \\
\hline true positive & 118 & 110 & 73 & 67 \\
\hline true negative & 309 & 302 & 321 & 316 \\
\hline false positive & 16 & 23 & 4 & 9 \\
\hline false negative & 21 & 29 & 66 & 72 \\
\hline sensitivity & $84.9 \%$ & $79.1 \%$ & $52.5 \%$ & $48.2 \%$ \\
\hline specificity & $95.1 \%$ & $92.9 \%$ & $98.8 \%$ & $97.2 \%$ \\
\hline $\begin{array}{l}\text { positive predictive } \\
\text { value }\end{array}$ & $88.1 \%$ & $82.7 \%$ & $94.8 \%$ & $88.2 \%$ \\
\hline $\begin{array}{l}\text { negative predictive } \\
\text { value }\end{array}$ & $93.6 \%$ & $91.2 \%$ & $82.9 \%$ & $81.4 \%$ \\
\hline
\end{tabular}

\section{T2w DIR-TSE edema imaging}

Overall, T2w DIR-TSE imaging revealed myocardial edema within the infarct zone in 24 patients (82.7\%) during the first blinded evaluation and in 21 patients (72.4\%) during the second image analysis. Combining both blinded evaluations resulted in a sensitivity of $50.4 \%$, a specificity of $98.0 \%$, a positive predictive value of $91.5 \%$, and a negative predictive value of $82.2 \%$ for the segment-based detection of myocardial edema within the infarct zone. Detailed results of the blinded reading are given in - Table 2. Statistical analysis revealed a good intra-rater agreement concerning the segmental rating of myocardial segments as edematous or non-edematous ( $\mathrm{K}=0.76$; $95 \%$ confidence interval 0.68 to 0.84 ). Combining both blinded evaluations resulted in a mean edema score of $9.7 \pm 7.8$, whereas the observed T2w DIRTSE edema scores were considerably lower compared to the observed LGE score $(9.6 \pm 7.6$ vs.15.3 $\pm 7.0, \mathrm{p}<0.0001 ; 9.7 \pm 7.8$ vs. $15.3 \pm 7.0, \mathrm{p}<0.0001)$. Image artifacts were observed in 100 of 390 (25.6\%) acquired T2w DIR-TSE, and 24 LV segments were rated as not evaluable due to non-diagnostic image quality.

\section{T2 mapping vs. T2w DIR-TSE}

Compared to the current standard for myocardial edema imaging (T2w DIR-TSE), T2 mapping depicted myocardial edema in a considerably higher number of LV segments in both analyses (131 vs. $78, \mathrm{p}<0.0001 ; 134$ vs. 76, $\mathrm{p}<0.0001)$. Significantly higher truepositive ( 118 vs. $73, \mathrm{p}<0.0001 ; 110$ vs. $76, \mathrm{p}<0.0001$ ) and falsepositive results ( 16 vs. $4 ; \mathrm{p}=0.037 ; 29$ vs. $9, \mathrm{p}=0.033$ ), as well as significantly lower false-negative ( 21 vs. $66, \mathrm{p}<0.0001 ; 29$ vs. 72 ; $\mathrm{p}<0.0001$ ) and true-negative results (309 vs. 321, $\mathrm{p}=0.031 ; 302$ vs. 316, $\mathrm{p}=0.024$ ) were observed during segmental edema analyses for T2 mapping compared to T2w DIR-TSE. The edema scores derived from T2 mapping were significantly higher compared to the edema scores derived from the T2w DIR-TSE images in both analyses $(17.7 \pm 8.0$ vs. $9.6 \pm 7.6, \mathrm{p}<0.001 ; 17.8 \pm 9.1$ vs. $9.7 \pm 8.2$, $\mathrm{p}=0.0001)$. T2 mapping resulted in a significantly lower number of slices with image artifacts ( 3 vs. 100 / 0.6\% vs. $21.6 \%$, p = 0.001) and in a significantly lower number of LV segments that were rated as non-evaluable ( 0 vs. $24, \mathrm{p}=0.012$ ) compared to $\mathrm{T} 2 \mathrm{~W}$ DIR-TSE imaging.

\section{Discussion}

Even though T2 imaging of the myocardium has improved greatly during the past decades, myocardial edema imaging remains challenging in the clinical routine due to several limitations of the currently used T2-weighted dark-blood TSE sequences. These limitations include regional myocardial signal variation caused by phased array coils, bright rim artifacts from stagnant blood, and myocardial signal loss caused by through-plane motion [10-12]. Since several experimental and clinical studies have confirmed the great value of myocardial edema imaging in AMI $[7,19,20]$ as well as in inflammatory cardiomyopathies [9], great efforts have been made to overcome the limitations of T2w darkblood TSE imaging. Within the scope of these efforts, T2w brightblood SSFP sequences have been developed within the last years $[13,21,22]$. Even though these approaches have addressed several of the mentioned shortcomings, these sequences are not common in the clinical routine presumably due to the fact that most of these techniques tend to provide a lower contrast between edematous and remote myocardium compared to T2weighted dark-blood TSE images [23]. 


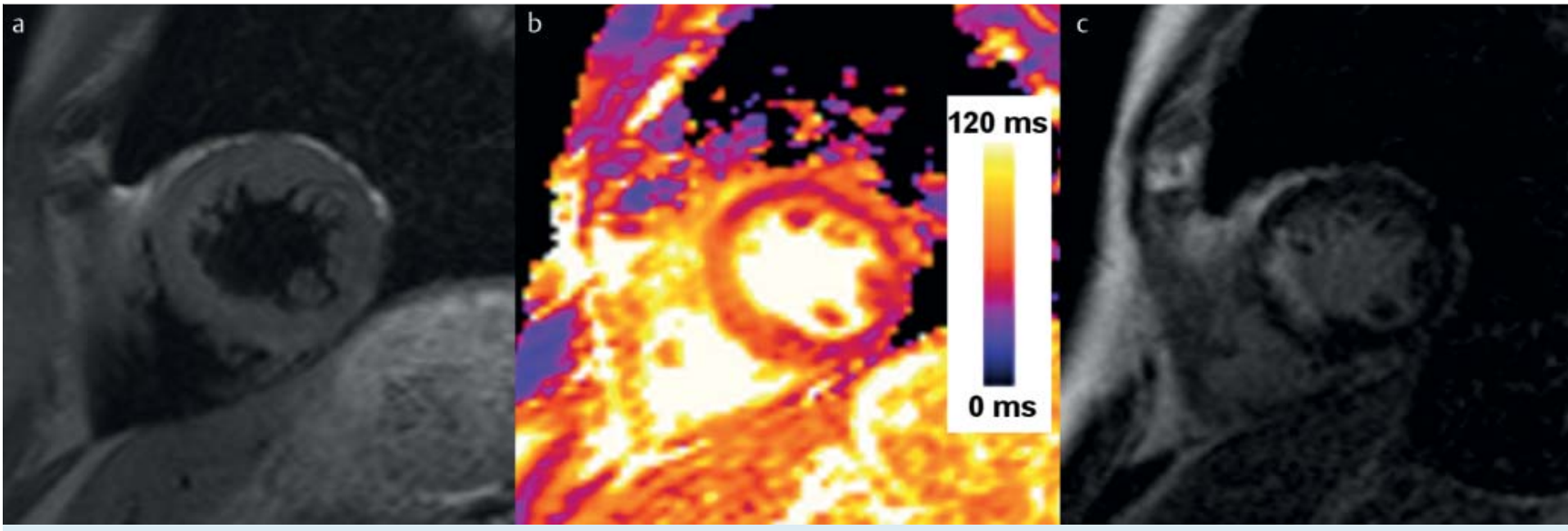

Fig. 1 T2w DIR-TSE a, T2 map b, and late gadolinium enhancement (LGE) short-axis image $\mathbf{c}$ of a 70-year-old male with ST-segment elevation myocardial infarction 2 days after percutaneous coronary intervention of the left anterior descending artery: Subendocardial infarction zone mid-myocardial septal with $50-75 \%$ transmurality c; consecutive myocardial edema that is visible only in the $\mathrm{T} 2 \mathrm{map} \mathbf{b}$.
Abb. 1 T2w-DIR-TSE a, T2-Map b und "Late Gadolinium Enhancement“ (LGE; c) Kurzachsen-Bilder eines 70-jährigen Patienten mit ST-HebungsMyokardinfarkt, 2 Tage nach perkutaner Revaskularisation des Ramus interventricularis anterior: Subendokardiale Infarktzone mittmyokardial septal mit 50 - 75\%iger Transmuralität c. Das konsekutive Myokardödem ist nur in der T2-Map sichtbar $\mathbf{b}$
Quantitative T2 mapping is a different approach for myocardial edema imaging that enables the differentiation of edematous from non-edematous myocardium by means of absolute values and not by differences in relative signal intensities. Even though multiple studies have reported myocardial T2 values over the past 25 years [24-26], T2 quantification has not been introduced into the clinical routine due to the fact that primarily spin-echobased techniques, which are time-consuming and prone to motion artifacts, were used.

As shown in the present study, the employed edema imaging technique, which combines T2w SSFP imaging with T2 mapping, enables reliable generation of myocardial T2 maps within a single breath-hold in the clinical routine. Compared to the currently accepted clinical standard for myocardial edema imaging - T2w dark-blood TSE $[8,9]$ - this T2 mapping technique shows a remarkably lower frequency of visual image artifacts and a significant reduction in the number of LV segments with non-diagnostic image quality. These findings are in good agreement with the initial results from Giri et al., who demonstrated in healthy volunteers and a rather small number of patients $(n=3)$ that this T2 mapping technique is much less sensitive to motion artifacts than TSE imaging and is not sensitive to artifacts from slow-flowing blood [14]. However, it must be critically noted that the visual analysis of image artifacts in color-coded maps is more difficult than the detection of image artifacts in T2w dark-blood TSE images, so that it cannot be excluded that the frequency of artifacts in the T2 maps was underestimated in the present study.

Comparison of the T2 mapping approach to T2w dark-blood TSE imaging showed a considerably higher sensitivity for the detection of myocardial edema in the infarct zone ( $82.0 \%$ vs. $50.4 \%$ ). Even though the most likely reason for this seems to be the fact that the utilized T2 mapping technique is much less sensitive to artifacts than TSE imaging, it also appears likely that visual assessment of color-coded T2 maps is easier than the reading of T2w dark-blood TSE images. While small alterations in the myocardial water content can be easily seen in T2 maps due to the chosen windowing of the color coding, in which small changes in the $\mathrm{T} 2$ values result in a distinct change in color, myocardial edema is more difficult to depict in T2w dark-blood TSE images due to the inherent low contrast between edematous and remote myocardium ( $\bullet$ Fig. 1 - 3).

Compared to previously published studies that found sensitivities of up to $100 \%$ [27] for the detection of myocardial edema in patients with AMI by T2w dark-blood TSE imaging, our reported sensitivity appears very low at first glance. On closer examination, however, it is evident that these differences are predominantly caused by different study designs. Whereas Friedrich et al. [27] reported a sensitivity of $100 \%$ on a per-patient basis, the diagnostic accuracy in the present study was calculated on a persegment basis, which enables a more detailed analysis. In a recently published study Payne et al. assessed the diagnostic performance of T2w dark-blood TSE for the detection of myocardial edema in AMI on a coronary artery perfusion territory basis. They found that T2w dark-blood TSE showed myocardial edema within the perfusion territory of the culprit coronary artery only in $61 \%$ of cases [22].

Even though T2 mapping showed better sensitivity than T2w dark-blood TSE imaging, the sensitivity of T2 mapping is not perfect. A possible explanation for the somewhat limited sensitivity of T2 mapping might be the fact that $\mathrm{T} 2$ values in microvascular obstruction (MO) and remote myocardium are very similar (58.7 $\pm 6 \mathrm{~ms}$ vs. $56 \pm 3.4 \mathrm{~ms}$ [15]), which results in the fact that in the case of MO only the margin of the infarct shows higher T2 values compared to the remote myocardium. Since the spatial resolution of the T2 mapping approach is comparatively low due to constraints in the patients' breath-holding capabilities, it is possible that in the case of extensive MO the thin non-MO infarct zone cannot be depicted due to partial volume effects.

Interestingly, the T2 mapping technique showed a slightly lower specificity than T2w dark-blood TSE imaging ( $94 \%$ vs. $98 \%$ ) due to a statistically significantly higher number of false-positive results. One possible reason for this might be our definition of the standard of reference: LV segments were only rated as affected by AMI if a regional wall motion abnormality and/or an area of LGE in an ischemic pattern was present in an LV segment within the perfusion territory of the culprit vessel. However, it cannot be excluded that myocardial edema is the only sign of ischemic injury at the infarct border [20], which might have disadvantaged T2 


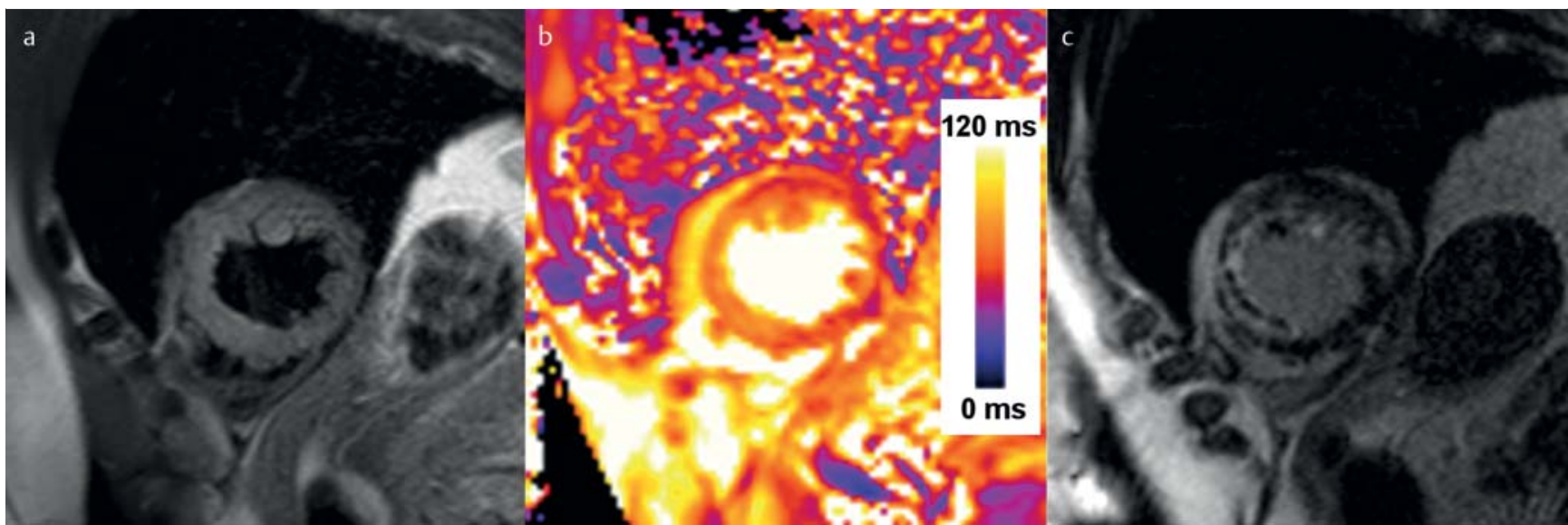

Fig. 2 T2w DIR-TSE image a, T2 map b, and late gadolinium enhancement (LGE) image $\mathbf{c}$ in short-axis orientation of a 57-year-old male with ST-segment elevation myocardial infarction 5 days after onset of clinical symptoms and percutaneous coronary intervention of the left anterior descending artery: Contrary to the T2w DIR-TSE image a, in which the myocardial edema is difficult to see, the T2 map clearly shows myocardial edema in the mid-ventricular septum b. Concordantly, an almost transmural myocardial infarction zone with a large no-reflow phenomenon is visible on the LGE image $\mathbf{c}$.
Abb. 2 T2w-DIR-TSE a, T2-Map b, und "Late Gadolinium Enhancement“ (LGE; C) Kurzachsen-Bilder eines 57-jährigen Patienten mit ST-HebungsMyokardinfarkt, 5 Tage nach dem Einsetzen klinischer Symptome und perkutaner Revaskularisation des Ramus interventricularis anterior: Im Gegensatz zum T2w-DIR-TSE Bild a, in dem das Myokardödem relativ schwierig abzugrenzen ist, zeigt die T2-Map deutlich ein Myokardödem im mittventrikulären Septum b. Übereinstimmend zeigt sich eine fast transmurale Infarktzone mit großem „no-reflow“-Phänomen im LGE Bild c.

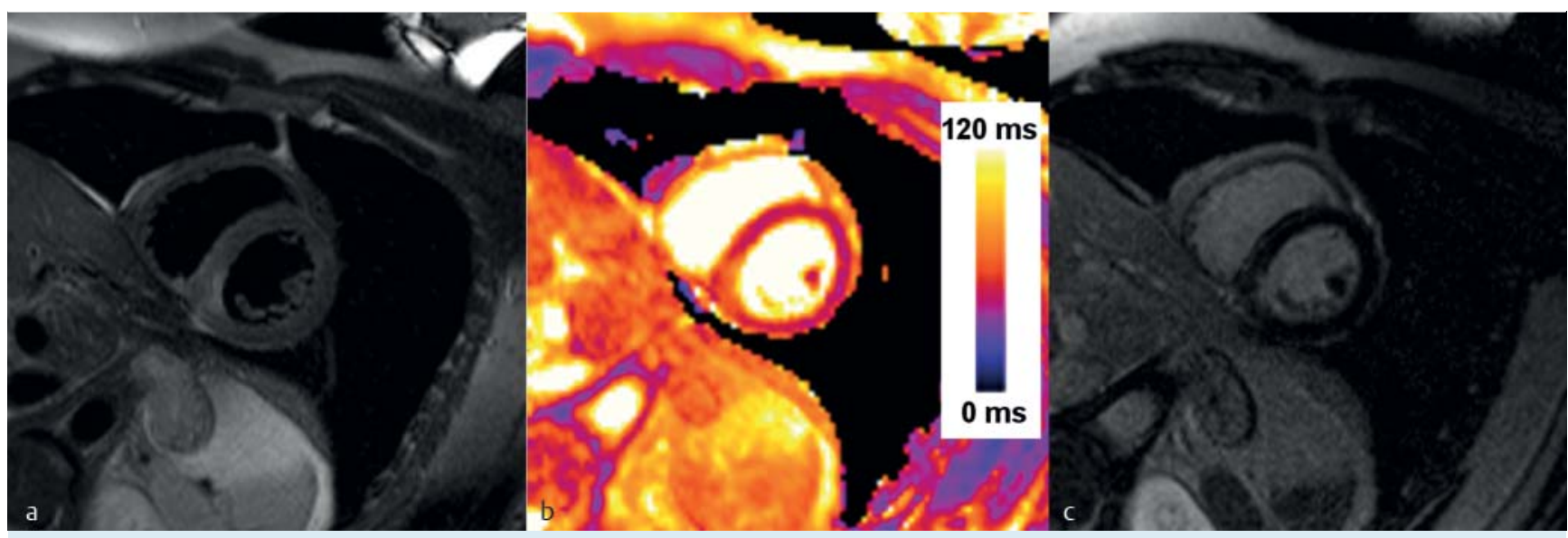

Fig. 3 T2w DIR-TSE a, T2 map b, and late gadolinium enhancement (LGE) short-axis image $\mathbf{c}$ of a 42-year-old male with ST-segment elevation myocardial infarction 3 days after percutaneous coronary intervention of the right coronary artery: Small subendocardial infarction zone mid-ventricular inferoseptal $\mathbf{c}$ with consecutive edema, which is clearly visible in the T2 map b as well as in the T2w DIR-TSE image a.
Abb. 3 T2w-DIR-TSE a, T2-Map b, und "Late Gadolinium Enhancement“ (LGE; c) Kurzachsen-Bilder eines 42-jährigen Patienten mit ST-HebungsMyokardinfarkt, 3 Tage nach perkutaner Revaskularisation der rechten Koronararterie: Es zeigt sich eine kleine, subendokardiale Infarktzone mittventrikulär inferoseptal c mit konsekutivem Ödem, welches sowohl in der T2-Map b als auch im T2w-DIR-TSE Bild a deutlich sichtbar ist. mapping due to its higher sensitivity. However, the difference in the observed specificities is small and insignificant from a clinical point of view.

Our semiquantitative scoring showed fairly similar values for the extent of myocardial edema as assessed by T2 mapping and the extent of myocardial infarction as assessed by LGE. However, from previous studies it could have been expected that the T2 map edema score would be higher than the LGE score, since it has been shown that the edema zone is larger than the LGE zone in AMI [22, 28, 29]. However, the conflict between our findings and this expectation is only contrived. Since T2 mapping was not able to detect myocardial edema in all LV segments affected by AMI, the observed similar overall T2 map edema and LGE scores indicate that in LV segments with visible edema the edema zone was in fact larger than the LGE zone.

In the present study only visual analysis of the T2 maps was performed to detect edematous myocardium. Even though this approach foregoes the opportunity for automated edema detection, which is an obvious option in T2 mapping due to its quantitative character, visual analysis of the T2 maps will in all probability be the most common approach for the interpretation of T2 maps in the clinical routine. Therefore, detailed analysis of this approach is of great clinical interest, possibly even more so than analysis of the performance of automated edema detection algorithms. However, automated edema imaging is of clinical interest, too. Unfortunately, several facts might hinder automated edema deli- 
neation in T2 maps, even though T2 mapping provides absolute values instead of relative signal intensities. As shown in the study by Giri et al. [14], the T2 mapping technique yields significantly higher T2 values in the apical segments compared to the basal and mid-ventricular segments when using short-axis T2 maps possibly due to partial volume effects - which might hinder the use of a fixed threshold. Moreover, the graphic presentation of the mean T2 values of the infarct zone and in the remote myocardium in patients with AMI provided in the study by Verhaert et al. [15] demonstrated a certain overlap between these T2 values which might also hinder automated delineation of myocardial edema. Nevertheless, the development and validation of software algorithms for the automated detection of edematous myocardium in T2 maps is an interesting, although nontrivial, challenge for further studies.

\section{Limitations}

$\nabla$

First of all, as mentioned above, the standard of reference used for the calculation of the diagnostic accuracy of both edema imaging approaches is not without limitation, which might have affected the calculated diagnostic accuracies. Moreover, since the present study focused on the diagnostic performance of T2 mapping in the clinical routine, it did not provide technical parameters like signal-to-noise ratios or $\mathrm{T} 2$ values of edematous and remote myocardium. However, these values have been investigated in detail previously [15], which relativizes this limitation.

\section{Conclusion}

$\nabla$

The present study shows that the novel T2 mapping technique employed here allows the visualization of myocardial edema in AMI with a high sensitivity and specificity. This technique offers significantly better diagnostic accuracy in terms of a higher sensitivity as well as more robust image quality compared to the current standard for myocardial edema imaging, which is T2w darkblood TSE imaging.

\section{References}

1 Abdel-Aty H, Tillmanns C. The use of cardiovascular magnetic resonance in acute myocardial infarction. Curr Cardiol Rep 2010; 12 : $76-81$

2 Wong DT, Richardson JD, Puri R et al. The role of cardiac magnetic resonance imaging following acute myocardial infarction. European radiology 2012; 22: $1757-1768$

3 Achenbach S, Barkhausen J, Beer $M$ et al. Consensus recommendations of the German Radiology Society (DRG), the German Cardiac Society (DGK) and the German Society for Pediatric Cardiology (DGPK) on the use of cardiac imaging with computed tomography and magnetic resonance imaging. Fortschr Röntgenstr 2012; 184: 345-368

4 Hergan K, Globits S, Schuchlenz $H$ et al. Clinical relevance and indications for cardiac magnetic resonance imaging 2013: an interdisciplinary expert statement. Fortschr Röntgenstr 2013; 185: 209-218

5 Abdel-Aty H, Simonetti O, Friedrich MG. T2-weighted cardiovascular magnetic resonance imaging. J Magn Reson Imaging 2007; 26: 452 459

6 Abdel-Aty H, Zagrosek A, Schulz-Menger J et al. Delayed enhancement and T2-weighted cardiovascular magnetic resonance imaging differentiate acute from chronic myocardial infarction. Circulation 2004; 109: $2411-2416$

7 Aletras AH, Tilak GS, Natanzon A et al. Retrospective determination of the area at risk for reperfused acute myocardial infarction with T2weighted cardiac magnetic resonance imaging: histopathological and displacement encoding with stimulated echoes (DENSE) functional validations. Circulation 2006; 113: 1865 - 1870

8 Kramer CM, Barkhausen J, Flamm SD et al. Standardized cardiovascular magnetic resonance imaging (CMR) protocols, society for cardiovascular magnetic resonance: board of trustees task force on standardized protocols. J Cardiovasc Magn Reson 2008; 10: 35

9 Friedrich MG, Sechtem U, Schulz-Menger J et al. Cardiovascular magnetic resonance in myocarditis: A JACC White Paper. J Am Coll Cardiol 2009; 53: 1475 - 1487

10 Keegan J, Gatehouse PD, Prasad SK et al. Improved turbo spin-echo imaging of the heart with motion-tracking. J Magn Reson Imaging 2006; 24: $563-570$

11 Pennell $D$. Myocardial salvage: retrospection, resolution, and radio waves. Circulation 2006; 113: 1821 - 1823

12 Arai $A E$. Using magnetic resonance imaging to characterize recent myocardial injury: utility in acute coronary syndrome and other clinical scenarios. Circulation 2008; 118: 795-796

13 Kellman P, Aletras AH, Mancini C et al. T2-prepared SSFP improves diagnostic confidence in edema imaging in acute myocardial infarction compared to turbo spin echo. Magn Reson Med 2007; 57: 891-897

14 Giri S, Chung YC, Merchant A et al. T2 quantification for improved detection of myocardial edema. J Cardiovasc Magn Reson 2009; 11: 56

15 Verhaert D, Thavendiranathan P, Giri S et al. Direct T2 quantification of myocardial edema in acute ischemic injury. JACC Cardiovasc Imaging 2011; 4: 269-278

16 Thygesen K, Alpert JS, White HD et al. Universal definition of myocardial infarction. Circulation 2007; 116: 2634-2653

17 Wagner A, Mahrholdt H, Thomson L et al. Effects of time, dose, and inversion time for acute myocardial infarct size measurements based on magnetic resonance imaging-delayed contrast enhancement. Journal of the American College of Cardiology 2006; 47: 2027-2033

$18 \mathrm{Kim} \mathrm{RJ,Wu} \mathrm{E,} \mathrm{Rafael} \mathrm{A} \mathrm{et} \mathrm{al.} \mathrm{The} \mathrm{use} \mathrm{of} \mathrm{contrast-enhanced} \mathrm{magnetic}$ resonance imaging to identify reversible myocardial dysfunction. N Engl J Med 2000; 343: 1445-1453

19 Abdel-Aty H, Schulz-Menger J. Cardiovascular magnetic resonance T2weighted imaging of myocardial edema in acute myocardial infarction. Recent Pat Cardiovasc Drug Discov 2007; 2: 63 - 68

20 Abdel-Aty H, Cocker M, Meek C et al. Edema as a very early marker for acute myocardial ischemia: a cardiovascular magnetic resonance study. J Am Coll Cardiol 2009; 53: 1194-1201

21 Aletras AH, Kellman P, Derbyshire JA et al. ACUT2E TSE-SSFP: a hybrid method for T2-weighted imaging of edema in the heart. Magn Reson Med 2008; 59: 229-235

22 Payne AR, Casey M, McClure J et al. Bright Blood T2 Weighted MRI Has Higher Diagnostic Accuracy Than Dark Blood STIR MRI for Detection of Acute Myocardial Infarction and for Assessment of the Ischemic Areaat-Risk and Myocardial Salvage. Circ Cardiovasc Imaging 2011

23 Viallon M, Mewton N, Thuny $F$ et al. T2-weighted cardiac MR assessment of the myocardial area-at-risk and salvage area in acute reperfused myocardial infarction: comparison of state-of-the-art dark blood and bright blood T2-weighted sequences. Journal of magnetic resonance imaging: JMRI 2012; 35: 328-339

24 Bottomley PA, Foster TH, Argersinger RE et al. A review of normal tissue hydrogen NMR relaxation times and relaxation mechanisms from 1$100 \mathrm{MHz}$ : dependence on tissue type, NMR frequency, temperature, species, excision, and age. Med Phys 1984; 11: 425 - 448

25 McNamara MT, Higgins CB, Schechtmann $N$ et al. Detection and characterization of acute myocardial infarction in man with use of gated magnetic resonance. Circulation 1985; 71: 717-724

26 Sparrow P, Amirabadi A, Sussman MS et al. Quantitative assessment of myocardial T2 relaxation times in cardiac amyloidosis. J Magn Reson Imaging 2009; 30: $942-946$

27 Friedrich MG, Abdel-Aty H, Taylor A et al. The salvaged area at risk in reperfused acute myocardial infarction as visualized by cardiovascular magnetic resonance. Journal of the American College of Cardiology 2008; $51: 1581-1587$

28 Stork A, Lund GK, Muellerleile $\mathrm{K}$ et al. Characterization of the peri-infarction zone using T2-weighted MRI and delayed-enhancement MRI in patients with acute myocardial infarction. Eur Radiol 2006; 16: $2350-2357$

29 Tilak GS, Hsu LY, Hoyt RF Jr et al. In vivo T2-weighted magnetic resonance imaging can accurately determine the ischemic area at risk for 2-day-old nonreperfused myocardial infarction. Invest Radiol 2008; 43: $7-15$ 